\title{
Computational Intelligence in Astronomy: A Survey
}

\author{
Ke Wang ${ }^{1}$, Ping Guo ${ }^{2 *}$, Fusheng $\mathrm{Yu}^{3}$, Lingzi Duan ${ }^{3}$, Yuping Wang ${ }^{4}, \mathrm{Hui} \mathrm{Du}^{5}$ \\ ${ }^{1}$ School of Computer Science and Technology, Beijing Institute of Technology, \\ Beijing, 100081, China \\ ${ }^{2}$ Image Processing and Pattern Recognition Laboratory, \\ School of System Science, Beijing Normal University, \\ Beijing, 100875, China \\ ${ }^{3}$ Laboratory of Mathematics and Complex Systems, Ministry of Education, \\ School of Mathematical Sciences, Beijing Normal University, \\ Beijing, 100875, China \\ ${ }^{4}$ School of Computer Science and Technology, Xidian University, \\ Xi'an, 710071, China \\ ${ }^{5}$ College of Computer Science and Engineering, Northwest Normal University, \\ Lanzhou, 730070, China
}

Received 28 April 2017

Accepted 5 January 2018

\begin{abstract}
With explosive growth of the astronomical data, astronomy has become a representative data-rich discipline so as to defy traditional research methodologies and paradigm to analyze data and discover new knowledge from the data. How to effectively process and analyze the astronomical data is a fundamental work while a key scientific requirement of modern astronomical surveys. This situation has motivated needs for fostering of a wide range of cooperation with the astronomers and computer scientists. Computational intelligence, an important research direction of artificial intelligence and information sciences, has been shown to be promising to solve complex problems in scientific research and engineering. This paper presents a review of the current state of the application of computational intelligence in astronomy. We believe that computational intelligence is expected to provide powerful tools for addressing challenges in astronomical data analysis.
\end{abstract}

Keywords: Computational intelligence; astronomical data analysis; neural networks; fuzzy set; evolutionary computation

*pguo@bnu.edu.cn 


\section{Introduction}

The technology advancement of observational instruments in astronomy will lead to a big bang of astronomical data. These data are typically high dimensional and multi-modal, which include multi-band spectra and images, various catalogs, time series data, and synthetic data. At the end of the last century, the total amount of image data of the Digitized Palomar Observatory Sky Survey (DPOSS) [1] was no more than 3 TB. By the beginning of this century, the data size of Sloan Digital Sky Survey (SDSS) [2] with multi-target fiber spectroscopy has reached $40 \mathrm{~TB}$. The forthcoming Large Synoptic Survey Telescope (LSST) will generate about 30 TB data at each observation night with an observation period of up to 10 years, and it is expected that 100,000 variable objects will be found every night. The total amount of LSST image data is estimated to reach about $70 \mathrm{~PB}$, the catalog will reach 10 20 $\mathrm{PB}$ [3].

Astronomical data big bang is mainly due to two factors: First, the advancement of technology has given observational instruments stronger capacity since 1970s. For example, the Keck telescope, at the top of Monaco in Hawaii, is the optical telescope with the largest aperture diameter of over 10 meters built in the world. The forthcoming Thirty Meter Telescope (TMT), which is being jointly built by multiple countries, has a larger diameter of 30 meters. These large telescopes have reached unprecedented level of observation width and depth. Secondly, with the development of CCD technology, astronomical data can be digitized directly into electronic documents, replacing the traditional means of digitizing by scanning negatives, which significantly improves the efficiency of data collection [4].

The large astronomical surveys have become the main forms and effective means of astronomers to study the universe. The richness of the data has brought new scientific opportunities to astronomy and severe challenges at the same time. Hence, the data-intensive era of astronomy necessitates a focus on automated, efficient and intelligential techniques and methodologies that can 'understand' certain tasks in astronomical research like astronomers and automatically mine large scale astronomical data for scientific discoveries, such as recognizing known objects, discovering unknown objects, search of rare objects and astronomical phenomenon. One way of performing such tasks is applying computational intelligence (CI) techniques. Generally speaking, computational intelligence is considered to be the family of scientific methods consisting of artificial neural networks, fuzzy systems, evolutionary computation, learning theory and probabilistic methods. Compared with other data processing methods, the most important advantage of CI is that it does not need to exactly establish the model of the problem and dose not requires prior knowledge but rather directly process the low level observed data (or only use inexact and incomplete knowledge) in nature-inspired computational methodologies and approaches. This feature is well suited to solve the complex problems of astronomical data analysis in which traditional methods or models are usually incompetent because of the lack of prior knowledge (e.g. the unknown or rare astronomical objects), or the data analysis involves uncertainties (e.g. the specious or incomplete data common in astronomy), or the data need to be processed in a stochastic way due to the complexity. We believe that CI will reinvent the study of modern astronomy. This is because of two separate factors. The first one is the fact that large scale astronomical data have become available thanks to the advent of astronomical instruments and observation technology. The second one includes improvements in software and hardware technologies, such as artificial intelligence, deep learning, and high-performance computing.

However, researchers including computer scientists, data scientists and astronomers have not yet to communicate effectively across specialties, to assimilate their achievements, and to consult with crossdisciplinary experts. We therefore review the current state of the application of computational intelligence in astronomy with the hope of giving a fairly comprehensive overview to the researchers from both computational intelligence and astronomy who are not very familiar with this new cross-discipline. We also hope that the review could provide inspiration for exciting new ideas and applications. The remainder of the paper summarizes the related application of computational intelligence techniques in astronomy from three aspects, i.e. the artificial neural network, the fuzzy set, and the evolutionary computation. We end this paper with the prospect for the future research in the last section. 


\section{Artificial neural networks in astronomy}

An artificial neural network (ANN) is a computational model inspired by biological neural networks and is used to approximate general nonlinear functions. ANN is a data-driven and self-adaptive computational intelligence technique. Unlike other parametric models, ANN does not have to make any prior assumptions about the underlying structure of the data and does not require any prior knowledge. Hence, it is easy to use and understand compared to other models, e.g., statistical methods. ANN could be used as a powerful tool to model complicated physical processes due to its ability to approximate any arbitrary nonlinear function.

Since the first astronomical application with ANN was presented in 1990 [5], the neural network has been the most widely used and well-known computational intelligence technique and machine learning model in astronomy [6]. Nowadays, ANN has been applied with success in many astronomical tasks, including but not limited to morphological classification of galaxies, evaluation of photometric redshifts, star/galaxy classification, stellar classification, stellar atmospheric parameters estimation, pulsar candidates identification.

\subsection{Morphological classification of galaxies}

Large-scale surveys e.g., SDSS and the forthcoming LSST, have generated or will generate vast amounts of images for millions of galaxies, and they will go on. Annotating these images with morphological information is a fundamental work because morphological information of galaxies can provide clues related to their formation and evolutionary history. However, this task cannot be done by unarmed human eye due to the huge data size. To classify galaxies by their morphology, a crowdsourcing project, Galaxy Zoo [7], was launched in 2007 in order to provide morphological classifications for nearly one million galaxies of SDSS. This project was made possible by combining the efforts from a large number of volunteers via a website. Unfortunately, as a result of the advent of astronomical instruments, new telescopes will generate tremendous amount of galaxy images, which makes crowdsourcing approach impractical in the future. This fact necessitates a focus on automated and scalable methods which are able to handle the task of morphological classification of galaxies.
In 1992, ANN was first applied in the morphological classification of galaxies. Storrie-Lombardi et al. [8] adopted a three layers feed-forward neural network to classify 3,517 galaxies into five classes, i.e., E, SO, $\mathrm{Sa}+\mathrm{Sb}, \mathrm{Sc}+\mathrm{Sd}$ and Irr with 1,700 galaxies as training samples. Since then, ANN has always been used as an important technique for the morphological classification of galaxies [9-15]. In this field, there are several issues that are worthy of consideration: 1) what is the network structure to be used? In the early works, the data sets are very small. Simple network structure, e.g. the basic single hidden layer feed-forward networks, are employed in the classification task. With the increase of data amount, it is possible to employ more complicated network structure with more learning capacity and less over-fitting risk. Recently, some deep learning models have been employed in morphological classification of galaxies. For instance, Dieleman et al. [14] use images from the Galaxy Zoo project to train a convolutional neural network (CNN) for galaxy morphology classification in the context of the Galaxy Challenge (https://www.kaggle.com/c/galaxy-zoo-the-galaxychallenge). Their model achieves state-of-the-art performance and wins the first place in the Galaxy Challenge. Subsequently, Huertas-Company et al. [15] extend this new methodology to high redshift galaxies by classifying $\sim 50,000$ galaxies with median redshift $\mathrm{z} \sim 1.25$. What are the features to be used as the input to the networks? Earlier works in this domain always use task specific parameters as the input to the networks, such as ellipticity (Ratio of the semi-major to semiminor axis length), area (number of pixels contained in the object) [16]. With the development of feature learning techniques, general features those are learned automatically in an unsupervised fashion have been utilized to galaxy morphology classification. This kind of feature learning method is applicable for big data scenario in the future. 3) How to improve the classification performance? Ensemble learning is a common used method to obtain better classification performance, which employs multiple learning algorithms. It is possible to combine multiple different ANNs or combine ANN and other classifiers into an ensemble one for better prediction performance. Ensemble learning has also been used in galaxy morphology classification $[9,11]$. 


\subsection{Photometric redshifts evaluation}

Redshift is a crucial cosmological parameter revealing the distance of galaxies according to the Hubble law. The photometric redshift evaluation is to use the broadband or medium-band photometry of galaxies to estimate their redshift. Although the distances are less accurate than those obtained with spectra, photometric redshift is very efficient compared with spectroscopic since the signal-to-noise in broad-band filters is much greater than the one in a dispersed spectrum and a whole field of galaxies may be imaged at once while spectroscopy is limited to individual galaxies [17]. Furthermore, the sheer number of objects with photometric measurements can often make up for the reduction in individual accuracy by suppressing the statistical noise of an ensemble calculation [6].

The fundamental approach to evaluate the photometric redshift is the template-fitting technique. For a given galaxy, the photometric redshift evolution is to find the redshift of the most closely matching template. This method is unintelligent and highly depending on the quality and representativeness of the template. Another method is the so-called empirical training set method, in which the redshift is predicted from the empirical data. It can be viewed as a regression task in the view of machine leaning. ANN is one of the most used empirical training set approaches [17-22]. In this kind of method, ANN usually takes fluxes in expert specific optical and near infrared filters as input and output the estimated redshift. It is worth noting that Hoyle [23] has tried to apply deep learning techniques to evaluate the photometric redshift. He uses the deep neural network to implement an end-to-end pipeline that takes the entire multi-band galaxy images as the input and removes the user intervention from the photometric redshift estimation.

\subsection{Star/galaxy classification}

Star/galaxy classification, also called star/galaxy separation or star/galaxy discrimination, is an old and long-standing problem and will pose challenges in future large imaging surveys. The basic task behind star/galaxy classification is to classify objects into stars and galaxies automatically using optical and near infrared images. ANN was first employed in star/galaxy classification task by Odewahn et al. in 1992 [24]. They train several different networks using 14-element image parameter set as the input and obtain a promising classification performance. Subsequently, ANN has always been used in this kind of task [25-30]. ANN in these works could be generally divided into two categories from the view of machine learning. The first one is the traditional supervised learning ANN, in which the true label of the training set is known. The second one is the unsupervised (or semi-supervised) learning ANN. For example, Miller and Coe [25] present a classifier using Kohonen self-organizing map (SOM). This approach requires much fewer labeled samples. Cortiglioni et al. [27] present a so-called direct image mode using SOM, in which objects are located and extracted from digitized plates as micro-images and these images are then directly used as inputs for subsequent processing.

With increasing quantities of image data produced by ongoing and forthcoming surveys, it is impractical to label the new data by experts intuitively. Therefore, the vast majority of new data are unlabeled. It's necessary to pay attention to the utilization of unlabeled data. From another point of view, star/galaxy classification is essentially a visual binary classification problem that is a research hotspot in computer vision and pattern recognition. Hence, new techniques or ideas of computer vision and pattern recognition community could be applied naturally in this task. For example, Kim et al. [31] recently present a star-galaxy classification framework that uses deep convolutional neural networks.

\subsection{Stellar classification}

Spectral types of stars provide valuable information in the study of the stellar physical parameters and the structure and evolution of the universe. The MK classification system [32] is the most frequently used one in stellar classification, in which letters $\mathrm{O}, \mathrm{B}, \mathrm{A}, \mathrm{F}$, $\mathrm{G}, \mathrm{K}$, and $\mathrm{M}$ are used to denote different spectral type of stars with different spectral characteristics. Each spectral type is then classified into 10 sub-class denoted by numeric digits from 0 to 9 . For the small data set, MK classification can be conducted by experts by comparing the stars to be classified with the standard ones. For large scale data set, this native way is infeasible and automated approaches are highly required. ANN has been used as a common tool to perform the classification task since it was first used in 1994 [33]. In ANN based stellar classification task, the input vectors to the networks are expert specific features or 
automatically extracted ones. For example, Navarro et al. [34] take a set of spectral indices as the input to the networks. In large spectroscopic survey, automated spectral feature extraction is a crucial procedure in stellar classification since extracting features manually is impractical. In general cases, principal component analysis (PCA) is employed as the feature extraction or dimensionality reduction method [35-37]. However, it can only represent the linear relations within the spectral data. Subsequently, nonlinear methods are introduced in the feature extraction in stellar classification tasks. Among these methods, locally linear embedding (LLE) has been a representative method applied in stellar feature learning [38, 39]. Recently, Wang et al. [40] adopt the deep neural network with a fast learning algorithm to learn features from stellar spectra automatically. Besides stellar classification with spectra, stars could also be classified with their images. Kuntzer et al. [41] train an ANN using single-band images of the diffraction shape of stars to estimate the stellar spectral type. Additionally, other models have been applied in stellar classification, such as gaussian classifier [42, 43], support vector machine (SVM) [44-46], and mixture models [47-52]. These models can also be viewed as neural networks essentially.

\subsection{Stellar atmospheric parameters estimation}

The stellar parameters estimation is to obtain the atmospheric parameters from the stellar spectra. These parameters, such as Teff (the effective temperature), log $\mathrm{g}$ (the surface gravity) and $[\mathrm{Fe} / \mathrm{H}]$ (the metallicity) reflect the intrinsic physical properties of stars, such as ages, masses, and elemental abundances. From the view of machine learning, this task can be regarded as a regression, in which the representative spectra along with known parameters are used to train a nonlinear regression model. This model is then used to predict the unknown stellar atmospheric parameters. Since ANN is a commonly used model for regression, it also can be used in stellar parameters estimation. Gulati et al. [53] train an ANN with synthetic spectra to assign the effective temperatures for G-K dwarfs. Bailer-Jones et al. [54] employ ANNs to produce physical parameters for observed stellar spectra. They generate a grid of synthetic spectra for a range of different atmospheric parameters. These synthetic optical stellar spectra are used to train a network. The trained network is then used to determine the effective temperature for over
5,000 real observed dwarfs and giants. Subsequently, Bailer-Jones [55] investigates the performance of ANN in the stellar parameter estimation with spectra at low resolution and SNR. It is found that neural networks can yield good determination of stellar parameters even at low resolution. Manteiga et al. [56] take coefficients of Fast Fourier Transform (FFT) and Discrete Wavelet Transform (DWT) as input features to an ANN which is trained to estimate the parameters Teff, $\log \mathrm{g},[\mathrm{Fe} / \mathrm{H}]$ and $[\alpha / \mathrm{Fe}]$. With the advancement of observation instruments, the increasing amount spectral data make the training of more sophisticated networks feasible. Yang and Li [57] use the auto-encoder to learn a set of local representations from stellar spectra. Then they use a back-propagation (BP) network to estimate stellar parameters.

\subsection{Pulsar candidate selection}

Searching for pulsars with modern radio telescopes, such as Parkes Multibeam Pulsar Survey (PMPS) [58], High Time Resolution Universe (HTRU) [59], or the Five-hundred meter Aperture Spherical Telescope (FAST) [60], is a significant and hot research area in astronomy. Raw data collected from radio telescopes are processed with techniques including radio frequency interference removal and de-dispersion, and then folded as pulsar candidates in the form of diagnostic plots and summary statistics. Traditionally, these pulsar candidates are further inspected by human experts on the plots and statistics to determine their authenticity. As the number of pulsar candidates and data volumes are increasing exponentially with the improvement of radio surveys, automatic pulsar candidate selection with machine learning algorithms, especially ANN, become popular recently. A lot of related works have been proposed for this task.

Eatough et al. [61] proposed to select pulsar candidates with three layers artificial neural networks, which consists of an input layer, a hidden layer and an output layer. The candidates are first preprocessed and represented by twelve feature scores. These 12 scores include 8 scores proposed in Keith et al. [62] and 4 scores proposed in this work. Then, these scores are regarded as inputs. In their architecture, the hidden layer has the same number of neurons as the input layer and the final layer has two outputs, indicating probabilities of the candidate being a credible pulsar or non-pulsar. By adopting 8 or 12 scores, Eatough et al. trained two 
architectures. With the trained artificial neural networks, they discovered a previously unidentified pulsar from a re-analysis of the PMPS.

Bates et al. [63] added some new feature scores as an improvement of the work of [61] and [62], resulting in 22 feature scores for each pulsar candidate. Then they trained a three fully connected layers ANN comprising 22 input neurons, 22 hidden neurons and 2 outputs. Their architecture was estimated to have an accuracy of $85 \%$ on validation set. In the mid-latitude portion of the HTRU, 75 pulsars were discovered with their method. They also argued that more representative pulsars are needed to improve the selection accuracy, and that different ANN models should be trained for selecting different kinds of pulsars.

Morello et al. [64] proposed SPINN (Straightforward Pulsar Identification using Neural Networks), in which a customized ANN is trained on 1,196 positive observations of pulsars and 90,000 negative observations from the southern HTRU survey. SPINN succeeds in finding all known real pulsars in the HTRU survey data and maintains a low false positive rate of $0.64 \%$. SPINN discovered four new pulsars in a reprocessing of the intermediate Galactic latitude area of HTRU.

Zhu et al. [65] proposed a system called Pulsar Imagebased Classification System (PICS). In this PICS, the prediction results of SVMs, ANNs and CNNs are combined together with a logistic regression function. Four features of the pulsar candidates are down-sampled or interpolated to a uniform size. In their work, summed profile is represented by a 64-bin vector, time vs. phase plot and frequency vs. phase plot are regarded as 2dimensional matrices (images), and dispersion-measure curve is a 60-bin vector. In all previous works, SVMs and ANNs just act as a discriminator to decide if the sample is a real pulsar or not. That is, handcrafted feature extraction is required in preprocess step before training the SVM and ANN. However, CNNs directly take the 2-dimension images as input and predict the results in an end-to-end way. The training candidates are gathered from Pulsar Arecibo L-band Feed Array (PALFA) survey. For each classifier, the performance of proposed CNN architecture in their work is superior to that of SVMs or ANNs on the Green Bank North Celestial Cap survey validation set. The system worked with Pulsar Arecibo L-band Feed Array (PALFA) survey and achieved six new pulsar discoveries.

Guo et al. [66] proposed a novel framework which combines deep convolution generative adversarial network (DCGAN) with SVM to address the imbalance data issue. DCGAN is used as the sample generation and discriminative feature learning model while SVM is employed as the classifier of pulsar candidates. Experimental results on two pulsar datasets illustrate the effectiveness and efficiency of this method.

\subsection{Others}

In addition to the applications mentioned above, ANN has also been applied in many other astronomical data analysis tasks, such as the study of solar activity detection [67], source detection in astronomical images [68], the classification of gamma-ray bursts [69], the removing of cosmic-ray in CCD images [70], the weak lensing measurement [71], the identification and study of Gravitational Wave [72, 73].

\section{Fuzzy set theory in astronomy}

Fuzzy set theory is developed to describe the linguistic expressions in daily life, such as tall, high, far etc. Unlike classical mathematics, it considers everything as a matter of degree, and uses membership degree to quantitatively describe the uncertainty. In the last few decades, fuzzy set theory has been applied to many fields, e.g. artificial intelligence, pattern recognition, control engineering, decision theory, expert system and so on. As the theory imitates the thinking pattern of human being, it's easy to comprehend and is suitable for many realistic problems.

Fuzzy set theory has been applied in many fields in astronomical data analysis since 1980s. It was first applied in the analysis and prediction of solar activity [74], using the technique of fuzzy clustering. With the development of fuzzy methods, more and more successful applications in astronomy tasks appeared, including the analysis and prediction of solar activity, classification tasks, telescope control system, celestial navigation and so on.

\subsection{Classification}

Classification has always been an important issue in astronomy. The large amount of data produced by 
photographic sky surveys require automated reduction techniques in order to extract information within reasonable times. Therefore, the automatic classification methods are urgently needed. Since the definitions and classification in astronomy are not such clear and the data are usually noisy in real surveys, fuzzy set theory is supposed to be an useful tool in this area. By contrast with other classification methods, e.g. the aforementioned ANN based ones, fuzzy set theory based methods employ degrees of memberships in the classification and recognition tasks. This characteristic is meaningful in astronomical scenarios. For example, stars from contiguous MK spectral type, e.g. A9 and F0, might have very similar features, hence it is reasonable to assess their memberships to different spectral types rather than using boolean true or false to arbitrarily classify them into certain categories. For another example, unexpected noise might generate fake emission lines in a spectrum while a true emission line might be weakened by the extinction or sky subtraction errors. Therefore, it is necessary to handle these uncertainties in the classification.

G. Spiekermann [75] first applied fuzzy set theory in classification of the faint galaxies in 1992. He developed a fully automated morphological classification system with a two-step classifier. In the first part, fuzzy set theory is used to determine the membership to early and late type galaxies. In the second part, Hubble equivalent types are extracted by applying heuristic methods to the results from the first part. The test results show the effectiveness in automated classification. Then Dumitrescuet al. [76] used fuzzy hierarchical clustering to classify pulsating stars through the shape of their light curves, and finally got the groups and their possible structure. However, Mähönen et al. [27] found the fuzzy classifier was slightly weaker than neural network and self-organized map in the star-galaxy classification. But they also pointed out that fuzzy classifier can describe how strongly the object belongs to this class through the output membership values. For galaxy classification in large survey, Coppa et al. [77] put forward a statistical and portable classification method making use of fuzzy clustering, which can operate on different datasets.

Another important application of fuzzy set theory is to classify stellar spectra. This was always done by experts with no help than their own experience in the past, which is obviously time-consuming and costly. Thus Rodríguez et al. [78] developed an automated knowledge-based analysis and classification system combining signal processing, expert systems and fuzzy logic techniques. They also found that expert systems with fuzzy logic are more suitable for the classification of the star's global temperature although all the techniques reach a success rate around $90 \%$. Manteiga et al. [79] also built a fuzzy logic knowledge-based system and proved that the success rate can go higher if errors in few spectral subtypes are considered acceptable as internal error. Whereas, Zhang et al. [80] chose fuzzy C-means clustering to build a soft discretization method of celestial spectrum characteristic line, in which fuzzy set theory was used to improve its anti-noise ability. With more techniques appear, researchers have tried more complex methods for stellar spectra classification, such as the manifold fuzzy twin support vector machine (Liu et al. [81]).

Fuzzy set theory has also been applied in other classification problems. For example, Freistetter [82] used fuzzy logic to classify near-earth-asteroids and Wegner [83] studied the two components of the double galaxy cluster based on fuzzy clustering.

\subsection{Solar activity}

As solar activity has a huge impact on Earth's ionosphere, climate, magnetic field and space activities, the analysis and prediction of it have always been hot issues in astronomy. The main task is to forecast the activity of the active regions as well as solar flares and coronal mass ejection (CME).

Since 17th century, people have already been observing and recording the sunspots and their movement. It's shown that sunspot activity is cyclical and is a basic sign of solar activity. Thus, early researches on the prediction of solar activity almost depended on the observation and analysis of sunspot groups. Considering that no one knows the mechanism of solar cycle, fuzzy set theory is quite suitable for the relevant problems. In 1985, Hu et al. [74] proposed a prediction method based on fuzzy classification of solar cycles, using the observed data of sunspots. Then other researchers also applied different fuzzy techniques on solar activity problems $[84,85]$.

With the development of telescopes, Extreme Ultraviolet Telescope (EIT) images appeared and had 
already been used to extract active regions. Fuzzy-based segmentation method shows better effect when processing solar images as the boundaries are not always well-defined and the images may be polluted with noise. K. Revathy et al. [86] proposed a fractalbased fuzzy technique to get the active regions. Then $\mathrm{V}$. Barra et al. [87] succeeded in automatically segmenting EUV solar images into Coronal Holes, Quiet Sun and Active Regions via a multichannel spatially constrained fuzzy clustering algorithm. They also improved the algorithm into a fast and robust one [88]. For images with noises, M. Druckmüller [89] put forward a noise adaptive fuzzy equalization method to process noisy images.

\subsection{Telescope control system}

Astronomical telescope plays an important role in the study of cosmic origin, galaxy distribution, discovery of new celestial bodies, exploration of the mysteries of the universe as well as the study of dark matter. How to control the speed and direction of the telescope accurately becomes an urgent problem when tracking an object. Since fuzzy logic can easily encode the linguistic information, it's quite a natural idea to apply fuzzy set theory by designing fuzzy logic controllers (FLC) based on operator experience.

Soliman et al. [90] designed the first FLC for the electric motor driver system of astronomical telescope in 1998. The controller utilized the position error and its rate of change to regulate the armature voltage of the DC motor drive of each link in the two-link model. And the controller contained two groups of fuzzy sets, both the motion and the control signal. The results demonstrated the superiority of FLC to the traditional PD fixed gain controller. Shen et al. [91] also used the position error to build FLC, with the idea of fuzzy controlling table to improve the efficiency. Then Attia [92] changed the static fuzzy sets into adaptive fuzzy sets, and improved the dynamic response of the overall system by improving the damping coefficient and decreasing the rise time and settling time. He subsequently put forward a model using hierarchical fuzzy controllers which improved the effectiveness through reduction of the rule base [93].

FLC has been applied to radio telescope. Su et al. [94] proposed a trajectory tracking strategy based on FLC and they added reference learning into the method later, making the system more robust. $\mathrm{Zi}$ et al. [95] added a proportional-integral controller in parallel instead to enhance the control performance for steady-state errors. Shao et al. [96] applied FLC for flexibly supported parallel manipulator and finally practiced on the Fivehundred-meter Aperture Spherical radio Telescope project.

\subsection{Others}

Besides the applications mentioned above, fuzzy set theory has also been applied in many other astronomical data analysis tasks, such as astronomical objects detection in wide-angle frames [97], cosmic-ray hit rejection [98], deriving photometric redshifts [99], stellar spectral outliers mining [100] and celestial navigation [101].

\section{Evolutionary computation in astronomy}

There are many optimization problems of high complexity related to handle a huge amount of data in astronomy and these so-called complex optimization methods are inherently limited by the size of the problem/data $[102,103]$. For instance, reliable analysis of large amounts of data is central to modern astrophysics and astronomical sciences in general. Evolutionary Algorithms include a group of heuristics based on the mechanism for the natural selection proposed by biology Darwinism and have been proven to be effective to the complex optimization problems. In evolutionary algorithms, a population of various individuals is evolved generation by generation where an individual is a solution to a problem and is encoded according to the necessities (e.g. it can be as simple as a binary array). The quality of an individual is measured by its fitness. The higher the quality of an individual, the higher its fitness [104]. From an astrophysics point of view, the applications of evolutionary computation in astronomy can be grouped into the following four fields.

\subsection{Study of distant bodies by observing anomalies on optical and radio astronomy}

One of the application areas of the evolutionary computation techniques in recent years is the search for planets with potential for life beyond our solar system. After the discovery of the first exoplanet orbiting the star 51-Pegasi b in 1995 [105], the techniques that permit indirect detection of these types of bodies have 
increased exponentially and the number of the planets discovered reaches 61 in the year 2009 and more than 3200 in total in 2016. In 1997, Lazio et al. used a basic genetic algorithm to fit Keplerian orbits and try to explain the irregularities in the periods of some pulsar by the presence of other secondary bodies [106], they observed the genetic algorithm was more efficient and found better solutions than the traditional simplex method and the simulated annealing method allowing the discovery of a planetary companion to the pulsar PSR B0329+54 and identified two other potential candidates: B1911-04 and B1929+10. In the stars, influence of planets can be measured via the Doppler shift to red or to blue in the spectrum of light that can reveal the presence of one planet, or two planets, or a complete planetary system. In 2010, Rozenkiewicz et al. [107] proposed a hybrid genetic algorithm combining a genetic algorithm with a simplex algorithm [108] to study star HD240210 which showed abnormalities that were not explained in the 1-planet Keplerian model but could be interpreted in context of the existence of an additional planet. The genetic algorithm was also used to explore different values for orbital parameters like orbital eccentricities, orbital periods, and time of periastron passage and eccentricity. In 1995, Charbonneau [109] proposed the implementation of a simple genetic algorithm but very customizable one (which made it ideal to be used in many physical applications of the field) [110], which later, became a reference point for many other works on curve fitting for galaxies rotation based on the orbital speed of stars and gas near the galactic center. This algorithm was refined several times in the next several years. Lazio and Cordes [111], proposed a possible method for searching for planetary systems around pulsars of known frequency by using a genetic algorithm to explore possible systems within a given acceptance values and margins by applying a mathematical model. Other particularly interesting work was carried out by Chwatal et al. in 2008 [112], and differs from the earlier ones and uses evolution strategies for the characterization of extra-solar planets. In 2012, F. Vachier et al. [113] implemented a new approach for determining the mutual orbits of directly-imaged multiple asteroids using a genetic-based algorithm. This approach was applied to several known binary asteroid systems (22 Kalliope, 3749 Balam, and 50000 Quaoar) observed with AO systems and HST. This statistical method is fast enough to permit the search for an orbital solution across a large parameter space and without a priori information about the mutual orbit. In 2014, S. Li et al. [114] proposed a general framework for the rapid design and optimization of low-thrust rendezvous/interception trajectories for future asteroid deflection missions.

\subsection{Stellar dynamic and helioseismology}

One of the first works where evolutionary computing techniques applied in study of stellar dynamic was proposed by Metcalfe in 1999 [115]. Due to the computational weights of calculating the light curves simulated using the matrix of parameters, the authors decide to implement a distributed system on a grid of twenty-five workstations with a central server where the genetic algorithm will organize the population and take control of work assignments. The proposed algorithm began generating a set of 1,000 arrays of randomly distributed parameters which are sent to the slave nodes to be responsible for calculating the theoretical curves according to the model UBV (The letters $\mathrm{U}, \mathrm{B}$, and V stand for ultraviolet, blue, and visual magnitudes) [116]. An interesting contribution is proposed by Ordonez et al. in 2010 [117], where a genetic algorithm is used to adjust the recognition fee of a neural network that handles the classification and characterization of stellar mass objects by studying their emission spectrum.

\subsection{Galaxies and other super-massive bodies}

In the early universe, star formation rate was higher than today, frequently colliding galaxies, generating a wealth of new stars with each encounter. Theis et al. in 1999 [110] used a modified version of the Charbonneau algorithm of 1995 [109] to study the evolutionary history of the galaxy NGC4449, a dwarf galaxy of Magellanic type are formed through its gravitational 
Table 1. The representative CI methods with applications in astronomy.

\begin{tabular}{|c|c|c|c|c|}
\hline Methods & & Applications & Advantages & Disadvantages \\
\hline ANN & $\begin{array}{l}\text { Single layer } \\
\text { feedforward } \\
\text { networks; } \\
\text { Multi- } \\
\text { layer Perception; } \\
\text { Stacked } \\
\text { Autoencoders; } \\
\text { SOM; } \\
\text { CNN; } \\
\text { GAN; } \\
\text { Hybrid networks; }\end{array}$ & $\begin{array}{l}\text { Morphological classification of } \\
\text { galaxies; } \\
\text { Photometric redshifts evaluation; } \\
\text { Star/galaxy classification; } \\
\text { Stellar classification; } \\
\text { Stellar atmospheric parameters } \\
\text { estimation; } \\
\text { Pulsar candidate selection; } \\
\text { Solar activity detection; } \\
\text { Astronomical objects detection; } \\
\text { gamma-ray bursts classification; } \\
\text { Cosmic-ray removing; } \\
\text { Weak lensing measurement; } \\
\text { Gravitational Wave signal } \\
\text { processing }\end{array}$ & $\begin{array}{l}\text { 1) Strong ability to approximate } \\
\text { any arbitrary nonlinear function. } \\
\text {;2) Data-driven and self-adaptive. } \\
\text { 3) Do not make prior } \\
\text { assumptions about the } \\
\text { underlying structure of the data } \\
\text { and do not require any prior } \\
\text { knowledge. } \\
\text { 4) Deep learning has been } \\
\text { applied in many fields with big } \\
\text { success as a powerful tool for the } \\
\text { end-to end feature learning. } \\
\text { 5) Hotspot of the research } \\
\text { community and rich open source } \\
\text { software toolkits. }\end{array}$ & $\begin{array}{l}\text { 1) The gradient descent } \\
\text { based training algorithms, } \\
\text { e.g. BP, suffer from a slow } \\
\text { training speed when the data } \\
\text { volume is large and/or the } \\
\text { network is deep. } \\
\text { 2) Black box with poor } \\
\text { interpretability. } \\
\text { 3) Unable to handle missing } \\
\text { value data. } \\
\text { 4) Difficult learning control } \\
\text { parameter adjustment and } \\
\text { tricky task of the design of } \\
\text { the network structure. }\end{array}$ \\
\hline
\end{tabular}

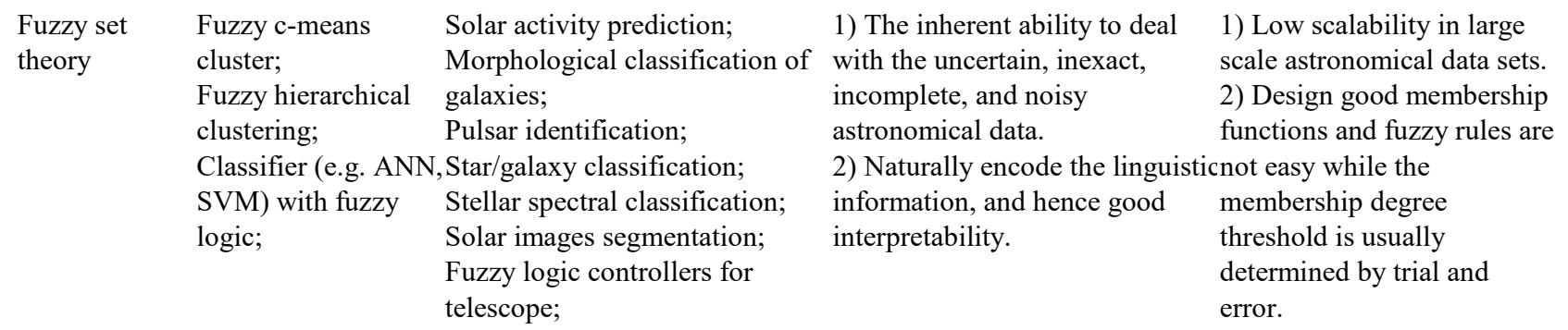

Astronomical objects detection;

Cosmic-ray removing;

Photometric redshifts evaluation;

Celestial Navigation

$\begin{array}{lll}\text { Evolutionary } & \text { Genetic algorithm; } & \text { Search and for planets with } \\ \text { computation } & \text { GA combined with } & \text { extraterrestrial civilization; } \\ & \text { ANN; } & \text { N-body problems; } \\ & \text { GA combined with } & \text { Orbit fitting; } \\ \text { simulated annealing } & \text { Characterize extra-solar planets; } \\ \text { algorithm; } & \text { Stellar dynamic; } \\ & \text { Galaxy evolution and } \\ & \text { characterization; } \\ & \text { Galaxy classification; } \\ & \text { Stellar population synthesis } \\ & \text { parameterization; } \\ & \text { gamma-ray burst detection; } \\ & \text { Gravitational waves detection; }\end{array}$

1) Strong ability to solve the 1) Slow convergence and complex optimization problems premature convergence. $\begin{array}{ll}\text { which are difficult to model } & 2 \text { ) Reliability and stability is }\end{array}$ and/or NP hard;

poor. However, the reliability and stability are complex astronomical processes among the key requirement or phenomena. for the scientific application, 3) Data-driven and require no such as astronomy. prior knowledge.

3) Low performance in high dimensional data that are common in astronomy.

In 2001, Wahde et al. [119] used a genetic algorithm to study the influence of past interaction between and the galaxy NGC5195 and the Messier M51 (commonly known as the Whirlpool Galaxy). Another remarkable work carried out by Cantu and Kamath in 2002 [120] was a hybrid solution AG-ANN, consisting of a genetic algorithm and back-propagation neural network with 
one hidden layer. In this paper, genetic algorithm performs the task of finding the best weights vector, and finds also the smallest number of input features to allow the neural network correctly classified observations of distant galaxies. For the galaxies composed of different kinds of stars, it is important to estimate the parameters of stellar population synthesis quickly and accurately. In 2015, Han [121] combined the genetic algorithm with the simulated annealing algorithm to estimate the parameters of stellar population synthesis.

\subsection{Supernovae and others high-energy events}

One example is the detection of a gamma-ray burst to 13 billion light years away by the NASA SWIFT observed in 2009 and confirmed the farthest supernova explosion recorded. This confirmed the study of Zwart et al. in 2001 [122]. In this work, they used an evolutionary algorithm for fitting a set of numerical models to several real observations of gamma emissions and tried to explain these by generation of high-energy emission jets in a rotation system where particles of ray interact with the interstellar medium. In the study of LIGO data analysis, many works have been done to suggest the use of different techniques of data mining. One of the most interesting works is the proposal of Lightman et al. at 2006 [123] which proposed the implementation of an evolutionary algorithm to maximize the chances of detecting gravitational waves and minimize the risk of false alarms in LIGO. The first paper using genetic algorithms to the analysis of data from LISA was introduced by Crowder and Cornish in 2006 [124]. It presents an effective method to isolate potential sources of gravitational waves from the possible tens of thousands of overlapping signals in the LISA data stream, reducing the level of confusing noise. The evolutionary approach is used as a first step in cleaning up the signal and constraining the parameters and allowing a further refining process using a Markov Chain and Monte Carlo algorithm.

\section{Summary}

In this paper, we intend to give a review of $\mathrm{CI}$ in astronomy, present a survey of the CI applications to various scientific problems refer to astronomy research field (see Table 1). Throughout, we emphasize $\mathrm{CI}$ as a tool to facilitate the scientific discovery, not as an end in itself, and to highlight areas where CI has been employed. We hope that the experts in CI could know enough about the scientific requirements of astronomy data processing and analysis. Meanwhile, astronomers would also need to realize such CI approaches could be an optional tool to solve the scientific problem they are addressing. We also hope that this paper could establish collaborative relationships for those experts in astronomy and $\mathrm{CI}$.

In recent years, areas related to CI has made significant progress and become a hot topic in the field of computer science. In the field of astronomy, it is noteworthy that many researchers have begun to try to apply emerging CI techniques to astronomical data processing and analysis, and carry out interdisciplinary research of CI and astronomy. For example, Graff et al. [125] provide a deep neural network toolkit for astronomy and apply it to three specific astronomical problems i.e. map of dark matter, identification of gamma-ray burster, and image compression for galaxies; Ravanbakhsh et al. [126] used generative adversarial networks (GAN) to generate distorted galaxy images due to gravitational lens effects for the study of dark matter. It can be expected that CI could make a difference in the future study of astronomy.

For the future study, we think new research efforts should focus on the following aspects: 1) faced with the astronomical data big bang, the scalability of the CI algorithm is a key point. New CI techniques with high scalability should be further studied. 2) Although the deep learning \& big data is the current mainstream model of artificial intelligence research, but in some specific astronomical problems, e.g. the discovery of rare objects, the available samples are still relatively scarce. Hence, methods for small data sets or imbalance data sets are still highly required. 3) Feature extraction is a crucial procedure in automated analysis of astronomical data. It determines the performance, efficiency, complexity and robustness of the subsequent algorithms. At present, the commonly used features of astronomical data processing are mainly extracted by traditional methods such as PCA, LLE and wavelet transform, or directly based on domain knowledge, such as line indices, colors and physical parameters. Meanwhile, deep learning models such as autoencoders, convolutional neural networks, generative adversarial networks have been shown to have more powerful feature learning capabilities, thus, application of these 
new methods in astronomical data feature learning is worthy of future researches. 4) How to interpret the results of astronomical data analysis with $\mathrm{CI}$ techniques, how to make use of domain knowledge in the process of data analysis to promote the performance of the CI algorithm are also worth pursuing in future research.

\section{Acknowledgements}

This work was fully supported by grants from the National Natural Science Foundation of China (61375045) and (11571001) and the Joint Research Fund in Astronomy (U1531242) under cooperative agreement between the National Natural Science Foundation of China (NSFC) and the Chinese Academy of Sciences (CAS). The section 2 is written by Fusheng $\mathrm{Yu}$ and Lingzi Duan, the section 3 is written by Yuping Wang and $\mathrm{Hui} \mathrm{Du}$, and other parts are written by $\mathrm{Ke}$ Wang and Ping Guo.

\section{References}

1. S. G. Djorgovski, R. R. Gal, R. R. De Carvalho, S. C. Odewahn, A. A. Mahabal, R. Brunner, P. Lopes, and D. Team, The Digital Palomar Observatory Sky Survey (DPOSS): General Description and the Public Data Release, Bulletin of the Astronomical Society of Brazil, vol. 34, no. 2, pp. 743, 2002.

2. D. G. York, J. Adelman, J. E. Anderson Jr, S. F. Anderson, J. Annis, N. A. Bahcall, J. Bakken, R. Barkhouser, S. Bastian, and E. Berman, The sloan digital sky survey: Technical summary, The Astronomical Journal, vol. 120, no. 3, pp. 1579, 2000.

3. K. D. Borne, A machine learning classification broker for the LSST transient database, Astronomische Nachrichten, vol. 329, no. 3, pp. 255-258, 2008.

4. C. Chenzhou;, X. Yanjie;, L. Jian;, Z. Yongheng;, L. Liang;, and C. Xiao, Virtual Observatory, an e-Science Environment for Astronomy, Bulletin of Chinese Academy of Sciences, vol. 28, no. 4, pp. 511-518, 2013.

5. J. Angel, P. Wizinowich, M. Lloyd-Hart, and D. Sandler, Adaptive optics for array telescopes using neural-network techniques, Nature, vol. 348, no. 6298, pp. 221-224, 1990.

6. N. M. Ball, and R. J. Brunner, Data mining and machine learning in astronomy, International Journal of Modern Physics D, vol. 19, no. 7, pp. 1049--1106, 2010.

7. M. A. Strauss, D. H. Weinberg, R. H. Lupton, V. K. Narayanan, J. Annis, M. Bernardi, M. Blanton, S. Burles, A. J. Connolly, and J. Dalcanton, Spectroscopic Target Selection in the Sloan Digital Sky Survey: The Main Galaxy Sample, Astronomical Journal, vol. 124, no. 3, pp. 1810-1824, 2002.
8. M. Storrie-Lombardi, O. Lahav, L. Sodre, and L. StorrieLombardi, Morphological classification of galaxies by artificial neural networks, Monthly Notices of the Royal Astronomical Society, vol. 259, no. 1, pp. 8-12, 1992.

9. J. De La Calleja, and O. Fuentes, Machine learning and image analysis for morphological galaxy classification, Monthly Notices of the Royal Astronomical Society, vol. 349, no. 1, pp. 87-93, 2004.

10. A. Naim, O. Lahav, L. Sodre, and M. Storrie-Lombardi, Automated morphological classification of APM galaxies by supervised artificial neural networks, Monthly Notices of the Royal Astronomical Society, vol. 275, no. 3, pp. 567-590, 1995.

11. D. Bazell, and D. W. Aha, Ensembles of classifiers for morphological galaxy classification, The Astrophysical Journal, vol. 548, no. 1, pp. 219, 2001.

12. M. Banerji, O. Lahav, C. J. Lintott, F. B. Abdalla, K. Schawinski, S. P. Bamford, D. Andreescu, P. Murray, M. J. Raddick, and A. Slosar, Galaxy Zoo: reproducing galaxy morphologies via machine learning, Monthly Notices of the Royal Astronomical Society, vol. 406, no. 1, pp. 342-353, 2010.

13. N. M. Ball, J. Loveday, M. Fukugita, O. Nakamura, S. Okamura, J. Brinkmann, and R. J. Brunner, Galaxy types in the Sloan Digital Sky Survey using supervised artificial neural networks, Monthly Notices of the Royal Astronomical Society, vol. 348, no. 3, pp. 1038-1046, 2004.

14. S. Dieleman, K. W. Willett, and J. Dambre, Rotationinvariant convolutional neural networks for galaxy morphology prediction, Monthly Notices of the Royal Astronomical Society, vol. 450, no. 2, pp. 1441-1459, 2015.

15. M. Huertas-Company, R. Gravet, G. Cabrera-Vives, P. G. Pérez-González, J. Kartaltepe, G. Barro, M. Bernardi, S. Mei, F. Shankar, P. Dimauro, and E. Bell, A Catalog of Visual-like Morphologies in the 5 CANDELS Fields Using Deep Learning, The Astrophysical Journal Supplement Series, vol. 221, no. 1, pp. 8, 2015.

16. D. Bazell, Feature relevance in morphological galaxy classification, Monthly Notices of the Royal Astronomical Society, vol. 316, no. 3, pp. 519-528, 2000.

17. A. E. Firth, O. Lahav, and R. S. Somerville, Estimating photometric redshifts with artificial neural networks, Monthly Notices of the Royal Astronomical Society, vol. 339, no. 4, pp. 1195-1202, 2003.

18. A. A. Collister, and O. Lahav, ANNz: estimating photometric redshifts using artificial neural networks, Publications of the Astronomical Society of the Pacific, vol. 116, no. 818, pp. 345, 2004.

19. O. Laurino, R. D'Abrusco, G. Longo, and G. Riccio, Astroinformatics of galaxies and quasars: a new general method for photometric redshifts estimation, Monthly Notices of the Royal Astronomical Society, vol. 418, no. 4, pp. 2165-2195, 2011.

20. Y. Zhang, L. Li, and Y. Zhao, Morphology classification and photometric redshift measurement of galaxies, 
Monthly Notices of the Royal Astronomical Society, vol. 392, no. 1, pp. 233-239, 2009.

21. E. Vanzella, S. Cristiani, A. Fontana, M. Nonino, S. Arnouts, E. Giallongo, A. Grazian, G. Fasano, P. Popesso, and P. Saracco, Photometric redshifts with the Multilayer Perceptron Neural Network: Application to the HDF-S and SDSS, Astronomy \& Astrophysics, vol. 423, no. 2, pp. 761-776, 2004.

22. C. Bonnett, Using neural networks to estimate redshift distributions. An application to CFHTLenS, Monthly Notices of the Royal Astronomical Society, vol. 449, no. 1, pp. 1043-1056, 2015.

23. B. Hoyle, Measuring photometric redshifts using galaxy images and Deep Neural Networks, Astronomy and Computing, vol. 16, pp. 34-40, 2016.

24. S. Odewahn, E. Stockwell, R. Pennington, R. Humphreys, and W. Zumach, Automated star/galaxy discrimination with neural networks, The Astronomical Journal, vol. 103, pp. 318-331, 1992.

25. A. Miller, and M. Coe, Star/galaxy classification using Kohonen self-organizing maps, Monthly Notices of the Royal Astronomical Society, vol. 279, no. 1, pp. 293-300, 1996.

26. S. Andreon, G. Gargiulo, G. Longo, R. Tagliaferri, and N. Capuano, Wide field imaging-I. Applications of neural networks to object detection and star/galaxy classification, Monthly Notices of the Royal Astronomical Society, vol. 319, no. 3, pp. 700-716, 2000.

27. F. Cortiglioni, P. Mähönen, P. Hakala, and T. Frantti, Automated star-galaxy discrimination for large surveys, The Astrophysical Journal, vol. 556, no. 2, pp. 937, 2001.

28. N. S. Philip, Y. Wadadekar, A. Kembhavi, and K. B. Joseph, A difference boosting neural network for automated star-galaxy classification, Astronomy \& Astrophysics, vol. 385, no. 3, pp. 1119-1126, 2002.

29. E. J. Kim, R. J. Brunner, and M. C. Kind, A hybrid ensemble learning approach to star-galaxy classification, Monthly Notices of the Royal Astronomical Society, vol. 453, no. 1, pp. 507-521, 2015.

30. M. T. Soumagnac, F. B. Abdalla, O. Lahav, D. Kirk, I. Sevilla, E. Bertin, B. T. Rowe, J. Annis, M. Busha, and L. Da Costa, Star/galaxy separation at faint magnitudes: application to a simulated Dark Energy Survey, Monthly Notices of the Royal Astronomical Society, vol. 450, no. 1, pp. 666-680, 2015.

31. E. J. Kim, and R. J. Brunner, Star-galaxy Classification Using Deep Convolutional Neural Networks, Monthly Notices of the Royal Astronomical Society, pp. stw2672, 2016.

32. W. Morgan, and P. Keenan, Spectral classification, Annual Review of Astronomy and Astrophysics, vol. 11, pp. 29, 1973.

33. T. Von Hippel, L. Storrie-Lombardi, M. StorrieLombardi, and M. Irwin, Automated classification of stellar spectra-I. Initial results with artificial neural networks, Monthly Notices of the Royal Astronomical Society, vol. 269, no. 1, pp. 97-104, 1994.
34. S. Navarro, R. Corradi, and A. Mampaso, Automatic spectral classification of stellar spectra with low signalto-noise ratio using artificial neural networks, Astronomy \& Astrophysics, vol. 538, pp. A76, 2012.

35. W. B. Weaver, and A. V. Torres-Dodgen, Accurate twodimensional classification of stellar spectra with Artificial Neural Networks, The Astrophysical Journal, vol. 487 , no. 2, pp. 847, 1997.

36. C. A. Bailer-Jones, M. Irwin, and T. Von Hippel, Automated classification of stellar spectra-II. Twodimensional classification with neural networks and principal components analysis, Monthly Notices of the Royal Astronomical Society, vol. 298, no. 2, pp. 361-377, 1998.

37. H. P. Singh, R. K. Gulati, and R. Gupta, Stellar spectral classification using principal component analysis and artificial neural networks, Monthly Notices of the Royal Astronomical Society, vol. 295, no. 2, pp. 312-318, 1998.

38. J. VanderPlas, and A. Connolly, Reducing the dimensionality of data: Locally linear embedding of sloan galaxy spectra, The Astronomical Journal, vol. 138, no. 5, pp. 1365, 2009.

39. S. F. Daniel, A. Connolly, J. Schneider, J. VanderPlas, and L. Xiong, Classification of stellar spectra with local linear embedding, The Astronomical Journal, vol. 142, no. 6, pp. 203, 2011.

40. K. Wang, P. Guo, and A. Luo, A new automated spectral feature extraction method and its application in spectral classification and defective spectra recovery, Monthly Notices of the Royal Astronomical Society, vol. 465, 2017.

41. T. Kuntzer, M. Tewes, and F. Courbin, Stellar classification from single-band imaging using machine learning, Astronomy \& Astrophysics, vol. 591, pp. A54, 2016.

42. Ping Guo, Yunde Jia, and Michael R. Lyu, A Study of Regularized Gaussian Classifier in High-Dimension Small Sample Set Case Based on MDL Principle with Application to Spectrum Recognition, Pattern Recognition, Vol. 41, No.9, pp.2842 2854, 2008.

43. Ping Guo, Spectral Pattern Recognition with Regularized Gaussian Classifier, Proceedings of IEEE International Conference on Neural Networks and Signal Processing, (ICNNSP'03), vol. 1, pp.727-730, 2003.

44. Fei Xing, Ping Guo, Stellar spectral recognition based on wavelet de-noising and SVM, Spectroscopy and Spectral Analysis, Vol.26, No.7 , pp.1368 1372, 2006 (in Chinese).

45. Ping Guo, Fei. Xing, and YuGang Jiang, Spectral Data Classification Using Supporting Vector Machine in Wavelet Domain, Proceedings of the IEEE Conference on Systems, Man and Cybernetics, pp.5894 5899, 2004.

46. Fei Xing, Ping Guo, Classification of Stellar Spectral Data Using SVM, in Advances in Neural Networks (ISNN2004), Fuliang Yin Jun Wang Chengan Guo (Eds.), Lecture Notes in Computer Science, Vol. 3173, pp.616-621, Springer-Verlag, Berlin Heidelberg 2004. 
47. Yugang Jiang, Ping Guo, Stellar Spectral Feature Extraction and Combination Analysis for Classification with ENN, Proceedings of 2005 International Conference on Machine Learning and Cybernetics (ICMLC2005), 2005 pp.3321-3326.

48. YuGang Jiang and Ping Guo, Mixture of Experts for Stellar Data Classification, Lecture Notes in Computer Science, Vol. 3497, pp.310-315, Springer-Verlag, Berlin Heidelberg 2005.

49. Ling Bai, Ping Guo, and ZhanYi Hu, Automated Stellar Classification for Large Surveys with EKF and RBF Neural Networks, The Chinese Journal of Astronomy and Astrophysics, Vol. 5, No. 2, pp.203-210, 2005.

50. Ling Bai,Ping Guo, A Novel Approach to Stellar Recognition by Combining EKF and RBF Net, in Advances in Neural Networks (ISNN2004), Fuliang Yin Jun Wang Chengan Guo (Eds.), Lecture Notes in Computer Science, Vol. 3173, pp.1002-1007, SpringerVerlag Berlin Heidelberg 2004.

51. YuGang Jiang, Ping Guo, Spectral Analysis and Recognition Using Multi-Scale Features and Neural networks, in Advances in Neural Networks (ISNN2004), Fuliang Yin Jun Wang Chengan Guo (Eds.), Lecture Notes in Computer Science, Vol. 3174, pp.369374, Springer-Verlag, Berlin Heidelberg 2004.

52. Ping Guo, Dongmei Qin, Zhanyi Hu, and Weimin Do, A Novel Method for Spectral Signal Pattern Recognition, Spectroscopy and Spectral Analysis, Vol.23, No.4, pp.811-815, 2003. (in Chinese).

53. R. Gulati, R. Gupta, and N. Rao, A comparison of synthetic and observed spectra for G-K dwarfs using artificial neural networks, Astronomy and Astrophysics, vol. 322, pp. 933-937, 1997.

54. C. A. Bailer-Jones, M. Irwin, G. Gilmore, and T. von Hippel, Physical parametrization of stellar spectra: the neural network approach, Monthly Notices of the Royal Astronomical Society, vol. 292, no. 1, pp. 157-166, 1997.

55. C. Bailer-Jones, Stellar parameters from very low resolution spectra and medium band filters, Astron. Astrophys, vol. 357, pp. 197-205, 2000.

56. M. Manteiga, D. Ordóñez, C. Dafonte, and B. Arcay, ANNs and wavelets: A strategy for gaia RVS low S/N stellar spectra parameterization, Publications of the Astronomical Society of the Pacific, vol. 122, no. 891, pp. 608, 2010.

57. T. Yang, and X. Li, An autoencoder of stellar spectra and its application in automatically estimating atmospheric parameters, Monthly Notices of the Royal Astronomical Society, vol. 452, no. 1, pp. 158, 2015.

58. R. N. Manchester, The Parkes multi-beam pulsar survey - I. Observing and data analysis systems, discovery and timing of 100 pulsars, Monthly Notices of the Royal Astronomical Society, vol. 328, no. 1, pp. 17-35, 2001.

59. S. Burke - Spolaor, M. Bailes, S. Johnston, S. D. Bates, N. D. R. Bhat, M. Burgay, N. D’Amico, A. Jameson, M. J. Keith, and M. Kramer, The High Time Resolution Universe Pulsar Survey - III. Single-pulse searches and preliminary analysis, Monthly Notices of the Royal Astronomical Society, vol. 416, no. 4, pp. 2465-2476, 2011.

60. R. NAN, D. LI, C. JIN, Q. WANG, L. ZHU, W. ZHU, H. ZHANG, Y. YUE, and L. QIAN, The five-hundred-meter aperture spherical radio telescope (FAST) project, International Journal of Modern Physics D, vol. 20, no. 06, pp. 989-1024, 2011.

61. R. P. Eatough, N. Molkenthin, M. Kramer, A. Noutsos, M. J. Keith, B. W. Stappers, and A. G. Lyne, Selection of radio pulsar candidates using artificial neural networks, Monthly Notices of the Royal Astronomical Society, vol. 407, no. 4, pp. 2443-2450, 2010.

62. M. J. Keith, R. P. Eatough, A. G. Lyne, M. Kramer, A. Possenti, F. Camilo, and R. N. Manchester, Discovery of 28 pulsars using new techniques for sorting pulsar candidates, Monthly Notices of the Royal Astronomical Society, vol. 395, no. 2, pp. 837-846, 2009.

63. S. D. Bates, M. Bailes, B. R. Barsdell, N. D. R. Bhat, M. Burgay, S. Burkespolaor, D. J. Champion, P. Coster, N. D'Amico, and A. Jameson, The High Time Resolution Universe Pulsar Survey - VI. An artificial neural network and timing of 75 pulsars, Monthly Notices of the Royal Astronomical Society, vol. 427, no. 2, pp. $1052-$ 1065, 2012.

64. V. Morello, E. D. Barr, M. Bailes, C. M. Flynn, E. F. Keane, and W. V. Straten, SPINN: a straightforward machine learning solution to the pulsar candidate selection problem, Monthly Notices of the Royal Astronomical Society, vol. 443, no. 2, pp. 1651-1662, 2014.

65. W. W. Zhu, A. Berndsen, E. C. Madsen, M. Tan, I. H. Stairs, A. Brazier, P. Lazarus, R. Lynch, P. Scholz, K. Stovall, S. M. Ransom, S. Banaszak, C. M. Biwer, S. Cohen, L. P. Dartez, J. Flanigan, G. Lunsford, J. G. Martinez, A. Mata, M. Rohr, A. Walker, B. Allen, N. D. R. Bhat, S. Bogdanov, F. Camilo, S. Chatterjee, J. M. Cordes, F. Crawford, J. S. Deneva, G. Desvignes, R. D. Ferdman, P. C. C. Freire, J. W. T. Hessels, F. A. Jenet, D. L. Kaplan, V. M. Kaspi, B. Knispel, K. J. Lee, J. v. Leeuwen, A. G. Lyne, M. A. McLaughlin, X. Siemens, L. G. Spitler, and A. Venkataraman, Searching for Pulsars Using Image Pattern Recognition, The Astrophysical Journal, vol. 781, no. 2, pp. 117, 2014.

66. P. Guo, F. Duan, P. Wang, Y. Yao. and X. Xin, Pulsar Candidate Identification with Artificial Intelligence Techniques. arXiv preprint arXiv:1711.10339, 2017.

67. R. A. F. Borda, P. D. Mininni, C. H. Mandrini, D. O. Gómez, O. H. Bauer, and M. G. Rovira, Automatic solar flare detection using neural network techniques, Solar Physics, vol. 206, no. 2, pp. 347-357, 2002.

68. M. Masias, J. Freixenet, X. Lladó, and M. Peracaula, A review of source detection approaches in astronomical images, Monthly Notices of the Royal Astronomical Society, vol. 422, no. 2, pp. 1674-1689, 2012.

69. A. Balastegui, P. Ruiz-Lapuente, and R. Canal, Reclassification of gamma-ray bursts, Monthly Notices of 
the Royal Astronomical Society, vol. 328, no. 1, pp. 283290, 2001.

70. W. Waniak, Removing cosmic-ray hits from CCD images in real-time mode by means of an artificial neural network, Experimental Astronomy, vol. 21, no. 3, pp. 151--168, 2006.

71. G. Nurbaeva, M. Tewes, F. Courbin, and G. Meylan, Hopfield neural network deconvolution for weak lensing measurement, Astronomy \& Astrophysics, vol. 577, pp. A104, 2015.

72. H. Kim, Y.-M. Kim, J. J. Oh, S. H. Oh, E. J. Son, and H. Chu, Deep Neural Networks for identifying noise transients in Gravitational-Wave Detectors, IAU General Assembly, vol. 22, pp. 57476, 2015.

73. D. George, and E. A. Huerta, Deep Neural Networks to Enable Real-time Multimessenger Astrophysics, arXiv preprint arXiv:1701.00008, 2016.

74. F. M. Hu, and M. H. Jiang, The fuzzy classification of the solar cycle and the prediction for the 22nd solar cycle, Chinese Journal of Space Science, vol. 5, pp. 46-51, 1988.

75. G. Spiekermann, Automated Morphological Classification of Faint Galaxies, Astronomical Journal, vol. 103, pp. 2102-2110, 1992.

76. A. Dumitrescu, A. Pop, and D. Dumitrescu, Structural Properties of Pulsating Star Light Curves Through Fuzzy Divisive Hierarchical Clustering, Astrophysics \& Space Science, vol. 250, no. 2, pp. 205-226, 1997.

77. G. Coppa, M. Mignoli, G. Zamorani, S. Bardelli, S. J. Lilly, M. Bolzonella, M. Scodeggio, D. Vergani, P. Nair, and L. Pozzetti, The bimodality of the 10k zCOSMOSbright galaxies up to $\mathrm{z} \sim 1$ : a new statistical and portable classification based on the optical galaxy properties, Astronomy \& Astrophysics, vol. 535, no. 2, pp. 773-777, 2011.

78. A. RodríGuez, B. Arcay, C. Dafonte, M. Manteiga, and I. Carricajo, Automated knowledge-based analysis and classification of stellar spectra using fuzzy reasoning, Expert Systems with Applications, vol. 27, no. 2, pp. 237244, 2004.

79. M. Manteiga, I. Carricajo, A. Rodríguez, C. Dafonte, and B. Arcay, Starmind: A Fuzzy Logic Knowledge-Based System for the Automated Classification of Stars in the MK System, Astronomical Journal, vol. 137, no. 2, pp. 3245-3253, 2009.

80. J. F. Zhang, X. Li, and H. F. Yang, A soft discretization method of celestial spectrum characteristic line based on fuzzy C-means clustering, Spectroscopy and Spectral Analysis, vol. 32, no. 5, pp. 1435, 2012.

81. Z. B. Liu, Y. Y. Gao, and J. Z. Wang, Automatic classification method of star spectra data based on manifold fuzzy twin support vector machine, Spectroscopy \& Spectral Analysis, vol. 35, no. 1, pp. 263266, 2015.

82. F. Freistetter, Fuzzy characterization of near-earthasteroids, Celestial Mechanics and Dynamical Astronomy, vol. 104, no. 1, pp. 93-102, 2009.
83. G. A. Wegner, The double galaxy cluster Abell 2465 - I Basic properties: optical imaging and spectroscopy, Monthly Notices of the Royal Astronomical Society, vol. 413, no. 2, pp. 1333-1352, 2011.

84. S. R. Zhou, G. L. Huang, Z. Z. Han, and C. Fang, The fuzzy forecast of the activity of solar active regions, Astrophysics and Space Science, vol. 280, no. 4, pp. 369380, 2002.

85. Z. Z. Han, and Y. H. Tang, Fuzzy set theory applied to CME importance classification, Chinese Astronomy \& Astrophysics, vol. 27, no. 3, pp. 303-307, 2003.

86. K. Revathy, S. Lekshmi, and S. R. P. Nayar, FractalBased Fuzzy Technique For Detection Of Active Regions From Solar Images, Solar Physics, vol. 228, no. 1, pp. 43-53, 2005.

87. V. Barra, V. Delouille, and J. F. Hochedez, Segmentation of extreme ultraviolet solar images via multichannel fuzzy clustering, Advances in Space Research, vol. 42, no. 5, pp. 917-925, 2008.

88. V. Barra, V. Delouille, M. Kretzschmar, and J. F. Hochedez, Fast and robust segmentation of solar EUV images: algorithm and results for solar cycle 23, Astronomy \& Astrophysics, vol. 505, no. 1, pp. 361-371, 2009.

89. M. Druckmüller, A Noise Adaptive Fuzzy Equalization Method for Processing Solar Extreme Ultraviolet Images, Astrophysical Journal Supplement, vol. 207, no. 207, pp. 25, 2013.

90. H. F. Soliman, A. F. A. Attia, M. A. L. Badr, A. M. Osman, and A. A. I. Gamaleldin, Fuzzy logic controller for the electric motor driving the astronomical telescope, in Astronomical Telescopes \& Instrumentation, 1998, pp. 415-424.

91. Shen, X. Chen, and Weimin, The Application of Fuzzycontrol in Astronomical Telescope Control System, Control \& Automation, 2005.

92. A. F. Attia, Adapted Fuzzy Controller for Astronomical Telescope Tracking, Experimental Astronomy, vol. 18, no. 1, pp. 93-108, 2004.

93. A. F. Attia, Hierarchical fuzzy controllers for an astronomical telescope tracking, Applied Soft Computing, vol. 9, no. 1, pp. 135-141, 2009.

94. Y. Su, B. Duan, B. Peng, and R. Nan, Fuzzy model reference learningfor tracking control of large radio telescope, ACTA AUTOMATICA SINICA, vol. 28, no. 6, pp. 1039-1042, 2002.

95. B. Zi, Z. C. Zhu, and J. L. Du, Analysis and control of the cable-supporting system including actuator dynamics, Control Engineering Practice, vol. 19, no. 5, pp. 491501, 2011.

96. Z. F. Shao, X. Tang, L. P. Wang, and Z. You, A fuzzy PID approach for the vibration control of the FSPM, International Journal of Advanced Robotic Systems, vol. 10, pp. 1, 2013.

97. L. Shamir, and R. J. Nemiroff, A Fuzzy Logic Based Algorithm for Finding Astronomical Objects in Wide- 
Angle Frames, Publications of the Astronomical Society of Australia, vol. 22, no. 2, pp. 111-117, 2005.

98. L. Shamir, A fuzzy logic-based algorithm for cosmic-ray hit rejection from single images, Astronomische Nachrichten, vol. 326, no. 6, pp. 428-431, 2005.

99. J. S. Speagle, and D. J. Eisenstein, Deriving Photometric Redshifts using Fuzzy Archetypes and Self-Organizing Maps. I. Methodology, Physics, vol. 124, no. 913, pp. 274-279, 2015.

100. Z. Liu, and W. Zhao, Study on Stellar Spectral Outliers Mining Based on Fuzzy Large Margin and Minimum Ball Classification Model, Spectroscopy and Spectral Analysis, 2016.

101. M. Ushaq, and J. Fang, A robust data fusion scheme for integrated navigation systems employing fault detection methodology augmented with fuzzy adaptive filtering, Proceedings of SPIE - The International Society for Optical Engineering, vol. 8916, no. 1, pp. 182-185, 2013.

102. B. Washbrook, and J. Li, The application of evolutionary computation to the analysis of the profiles of elliptical galaxies: a maximum likelihood approach. pp. 2607-2612 Vol. 3.

103. V. Rajpaul, Genetic algorithms in astronomy and astrophysics, Astrophysical Journal Supplement, vol. 101, no. 2, pp. 309, 2012.

104. E. Eberbach, Toward a theory of evolutionary computation, Biosystems, vol. 82, no. 1, pp. 1-19, 2005.

105. M. Mayor, and D. Queloz, A Jupiter-Mass Companion to a Solar-Type Star, Nature, vol. 378 , no. 6555 , pp. 355-+, 1995.

106. T. J. W. Lazio, Genetic Algorithms, Pulsar Planets, and Ionized Interstellar Microturbulence, Publications of the Astronomical Society of the Pacific, vol. 109, no. 739, pp. 1925, 1997.

107. A. Rozenkiewicz, K. Gozdziewski, and C. Migaszewski, Modeling radial velocities of HD 240210 with the Genetic Algorithms.

108. J. A. Nelder, and R. Mead, A Simplex Method for Function Minimization, Computer Journal, vol. 7, no. 4, pp. 308-313, 1965

109. P. Charbonneau, Genetic Algorithms in Astronomy and Astrophysics, Astrophysical Journal Supplement, vol. 101, no. 2, pp. 309, 1995.

110. C. Theis, Modeling Encounters of Galaxies: The Case of NGC4449, Proc. of the Annual Meeting of the Astronomische Gesellschaft, Heidelberg, 1999.

111. T. J. W. Lazio, and J. Cordes, Genetic Algorithms: Searching for Planets Around Pulsars, in Millisecond Pulsars: A Decade of Surprise, Aspen, Colorado, 1994.

112. A. M. Chwatal, and G. R. Raidl, Determining Orbital Elements of Extrasolar Planets by Evolution Strategies: Springer Berlin Heidelberg, 2007.

113. F. Vachier, J. Berthier, and F. Marchis, Determination of binary asteroid orbits with a genetic-based algorithm, Astronomy \& Astrophysics, vol. 543, no. 1, pp. 155-171, 2012.
114. S. Li, Y. Zhu, and Y. Wang, Rapid design and optimization of low-thrust rendezvous/interception trajectory for asteroid deflection missions, Advances in Space Research, vol. 53, no. 4, pp. 696-707, 2014.

115. T. S. Metcalfe, Genetic-Algorithm-based Light Curve Optimization Applied to Observations of the W UMa star BH Cas, Astronomical Journal, vol. 117, no. 5, pp. 2503, 2007.

116. H. L. Johnson, and W. W. Morgan, Fundamental stellar photometry for standards of spectral type on the revised system of the Yerkes spectral atlas, Astrophysical Journal, vol. 117, no. 3, pp. 313, 1953.

117. D. Ordóñez, C. Dafonte, M. Manteiga, and B. Arcay, Parameterization of RVS synthetic stellar spectra for the ESA Gaia mission: Study of the optimal domain for ANN training, Expert Systems with Applications, vol. 37, no. 2, pp. 1719-1727, 3, 2010.

118. M. Wahde, Determination of orbital parameters of interacting galaxies using a genetic algorithm. Description of the method and application to artificial data, Astronomy \& Astrophysics Supplement, vol. 132, no. 3, pp. 417-429, 1997.

119. M. Wahde, and K. J. Donner, Determination of the orbital parameters of the M 51 system using a genetic algorithm, Astronomy \& Astrophysics, vol. 379, no. 1, pp. 115-124, 2001.

120. E. Cantú-Paz, and K. Chandrika, Evolving neural networks for the classification of galaxies, in Conference on Genetic and Evolutionary Computation, 2002, pp. 1019-1026.

121. J. S. Han, Parameter Estimation of Stellar Population Synthesis Using A Combined Genetic Algorithm, Chinese Astronomy \& Astrophysics, vol. 39, no. 4, pp. 454-465, 2015.

122. Simon F. Portegies Zwart, and Tomonori Totani, Precessing jets interacting with interstellar material as the origin for the light curves of gamma - ray bursts, Monthly Notices of the Royal Astronomical Society, vol. 328, no. 3, pp. 951-957, 2001.

123. M. Lightman, J. Thurakal, J. Dwyer, R. Grossman, P. Kalmus, L. Matone, J. Rollins, S. Zairis, and S. Márka, Prospects of gravitational wave data mining and exploration via evolutionary computing, Journal of Physics Conference Series, pp. 58-65, 2006.

124. J. Crowder, N. J. Cornish, and J. L. Reddinger, LISA data analysis using genetic algorithms, Physical Review D Particles \& Fields, vol. 73, no. 73, pp. 348-353, 2006.

125. P. Graff, F. Feroz, M. P. Hobson, and A. Lasenby, SkyNet: an efficient and robust neural network training tool for machine learning in astronomy, Monthly Notices of the Royal Astronomical Society, vol. 441, no. 2, pp. 1741-1759, 2013.

126. S. Ravanbakhsh, F. Lanusse, R. Mandelbaum, J. Schneider, and B. Poczos, Enabling Dark Energy Science with Deep Generative Models of Galaxy Images, arXiv preprint arXiv:1609.05796, 2016., 2016. 